\title{
Spectral isomorphisms of Morse flows
}

\author{
by \\ T. Downarowicz (Wrocław), J. Kwiatkowski (Toruń) \\ and Y. Lacroix (Brest)
}

\begin{abstract}
A combinatorial description of spectral isomorphisms between Morse flows is provided. We introduce the notion of a regular spectral isomorphism and we study some invariants of such isomorphisms. In the case of Morse cocycles taking values in $G=\mathbb{Z}_{p}$, where $p$ is a prime, each spectral isomorphism is regular. The same holds true for arbitrary finite abelian groups under an additional combinatorial condition of asymmetry in the defining Morse sequence, and for Morse flows of rank one. Rank one is shown to be a spectral invariant in the class of Morse flows.
\end{abstract}

Introduction. Let $(X, T, \nu)$ be an ergodic measure preserving transformation of a standard probability space. Let $G$ be a compact abelian group with Haar measure $\lambda$. By a group extension over $T$ we shall mean the skew product $\left(X \times G, T_{\phi}, \nu \times \lambda\right)$, where $T_{\phi}(x, g)=(T(x), g \phi(x))$ with $\phi: X \rightarrow G$. The system $(X, T, \nu)$ is called a base and $\phi$ is a cocycle.

It is well known that the space $L^{2}(\nu \times \lambda)$ decomposes into the product of $T_{\phi}$-invariant subspaces $L_{\gamma}^{2}=\left\{f \otimes \gamma: f \in L^{2}(\nu)\right\}$ where $\gamma$ ranges over $\widehat{G}$. We will investigate spectral isomorphisms between pairs of group extensions by the same group $G$ over the same base $(X, T, \nu)$. We expect an isomorphism which leaves the subspaces $L_{\gamma}^{2}$ invariant to exhibit some "nice" properties. An isomorphism which sends each $L_{\gamma}^{2}$ to $L_{\widehat{v}(\gamma)}^{2}$, where $\widehat{v}$ is a group isomorphism of $\widehat{G}$, will have similar properties, because it can be easily reduced to one which leaves the spaces $L_{\widehat{v}(\gamma)}^{2}$ invariant (by applying to one of the cocycles the group automorphism $v$ of $G$ dual to $\widehat{v}$ ).

2000 Mathematics Subject Classification: 11K31, 37A30, 42A55, 94A99.

Key words and phrases: Morse sequence, spectral isomorphism.

Research of the first author supported by the grant KBN 2 P03A 03915 [98-01 r.].

Research of the second author supported by the grant KBN 2 P03A 00214.

The first draft of this paper was written while the first two authors were visiting the Université de Bretagne Occidentale, April-May 1998. 
Definition. We will say that a spectral isomorphism is regular if it sends each $L_{\gamma}^{2}$ to $L_{\widehat{v}(\gamma)}^{2}$, where $\widehat{v}$ is a group automorphism of $\widehat{G}$.

It has been proved in $[\mathrm{N}]$ and $[\mathrm{J}-\mathrm{L}-\mathrm{M}]$ that every spectral isomorphism induced by a metric isomorphism is regular (under the assumption that the base is a canonical factor of the group extensions).

The precise meaning of "nice" properties will become clear in the case of Morse flows, and it is discussed at the beginning of the section devoted to regular spectral isomorphisms. Roughly speaking, such isomorphisms provide a correspondence between certain $G$-valued functions, to which we no longer need to apply the characters, leading to purely combinatorial invariants and techniques.

Our main interest is in determining when two ergodic group extensions $T_{\phi}$ and $T_{\phi^{\prime}}$ over the same base are spectrally isomorphic and what kind of similarities this fact imposes on these flows. Thus, we feel free to choose from among all relevant spectral isomorphisms one which suits us best (for instance a regular one, if such exists) and treat all the remaining isomorphisms as "equivalent". We will do so in the following situation: suppose that the spectral types and multiplicities of $L_{\gamma_{1}}^{2}$ and $L_{\gamma_{2}}^{2}$ (with respect to the group extension $T_{\phi}$ ) coincide for some $\gamma_{1} \neq \gamma_{2} \in \widehat{G}$. Then, by the spectral theorem, there exists a spectral isomorphism $U$ of $L^{2}$ leaving the subspaces $L_{\gamma}^{2}$ invariant, except that it permutes $L_{\gamma_{1}}^{2}$ with $L_{\gamma_{2}}^{2}$. In such a case we can try to improve the regularity of the spectral isomorphism between $T_{\phi}$ and $T_{\phi^{\prime}}$ by composing it with the above isomorphism $U$.

In certain cases (e.g., for Morse cocycles, as we soon explain) we know that every spectral isomorphism sends each $L_{\gamma}^{2}$ to $L_{\widehat{\pi}(\gamma)}^{2}$, where $\widehat{\pi}$ is some permutation of the characters, a priori not necessarily a group automorphism. So far we know of no example of a pair of group extensions spectrally isomorphic via a permutation $\widehat{\pi}$ not equivalent to a group automorphism.

A better understanding of this phenomenon may be crucial in the investigations of certain spectral invariants for group extensions. A well known open problem is whether rank is a spectral invariant. One of the motivations for the present discussion is the discovery that rank one is preserved by spectral isomorphisms between Morse flows, mostly due to their regularity.

In this note we first characterize spectral isomorphism between Morse flows in terms of combinatorial properties of words obtained from the defining blocks via characters (Corollary 1 and Theorem 4). Next we introduce certain combinatorial characteristics (abstract autocorrelations) which then turn out to be invariant under regular spectral isomorphisms. Further, we describe three cases where every spectral isomorphism is regular (or equivalent to a regular one): 
(1) $G=\mathbb{Z}_{p}$ is the cyclic group of prime order,

(2) $G$ is an arbitrary finite group and one of the cocycles has an additional property AS,

(3) one of the Morse flows is of rank one.

In the first two cases we assume that the cocycles have "bounded lengths". Before handling the last case we provide a characterization of rank one within the class of Morse flows in terms of the formerly established regular invariants. Using this, in (3) we show that the other Morse flow is also of rank one.

The restrictions we had to make on Morse flows in (1) and (2) result from our computational techniques and we conjecture that they can be omitted. We also conjecture that all values of rank are preserved by regular spectral isomorphisms.

Due to the nature of the methods applied, we will concentrate mainly on the symbolic representation of Morse flows, rather than work in the cocycle setup.

Basics. Let us now introduce the basic definitions and facts: as usual, $\mathbb{Z}$ denotes the set of all integers, while $\mathbb{N}$ stands for $\{1,2,3, \ldots\}$. $\mathbb{T}$ denotes the unit circle on the complex plane, and $\bar{z}$ is the conjugate of $z$.

Let $G$ be a finite abelian group denoted multiplicatively, with unit 1 . As usual, we denote by $\widehat{G}$ the dual group of $G$, its elements (characters on $G$ ) are denoted by the letters $\gamma, \gamma^{\prime}$, etc., and $\gamma_{0}$ is the trivial character (i.e., the unit of $\widehat{G})$.

By a block $B$ of length $n \in \mathbb{N}$ over $G$ we mean a finite sequence $B=$ $\left(b_{0}, b_{1}, \ldots, b_{n-1}\right) \in G^{n}$. A sequence over $G$ has the form $B=\left(b_{0}, b_{1}, \ldots\right)$ $\in G^{\mathbb{N}}$. Sometimes, instead of $b_{i}$, we also write $B(i)$. We say that the block $B$ is symmetric if $b_{i}=b_{n-1-i}$ for each $0 \leq i<n$.

Definition 1. Let $B$ be a block of length $n$ over $G$. For $0 \leq k \leq n-1$ and $g \in G$ we define

$$
\operatorname{fr}_{B}(k, g)=\frac{1}{n} \#\left\{i: 0 \leq i \leq n-k-1,\left(b_{i}\right)^{-1} b_{i+k}=g\right\} .
$$

Clearly, $\operatorname{fr}_{B}(0, g)=1$ if $g=\mathbf{1}$ and 0 for other elements $g$.

Definition 2. Let $B=\left(b_{0}, b_{1}, \ldots, b_{n-1}\right) \in \mathbb{T}^{n}$ (such a $B$ will be called a word). The aperiodic autocorrelation function of $B$ is defined on $\{0,1, \ldots, n-1\}$ by

$$
\Phi_{B}(k)=\frac{1}{n} \sum_{i=0}^{n-k-1} \bar{b}_{i} b_{i+k}=\sum_{z \in \mathbb{T}} z \operatorname{fr}_{B}(k, z) .
$$

Clearly, $\Phi_{B}(0)=1$. 
If $A$ denotes a sequence over $G$ (or $\mathbb{T}$ ) then we define $\operatorname{fr}_{A}(k, g)$ (and $\left.\Phi_{A}(k)\right)$ as the limit of $\operatorname{fr}_{A_{n}}(k, g)$ (resp. $\Phi_{A_{n}}(k)$ ), where $A_{n}$ is the initial block in $A$ of length $n$. Of course, there is no guarantee that such limits exist.

Let $B=\left(b_{0}, b_{1}, \ldots, b_{n-1}\right)$ and $A=\left(a_{0}, a_{1}, \ldots, a_{m-1}\right)$ be two blocks over $G$. We define their product as the block $B \times A=\left(c_{0}, c_{1}, \ldots, c_{m n-1}\right)$, where

$$
c_{s+n t}=b_{s} a_{t}, \quad 0 \leq s<n, 0 \leq t<m .
$$

The above definition can also be applied to the case where $A$ represents a sequence over $G$.

The following lemma appears in many variants in the literature (see e.g. [D-L]) and it says that the autocorrelation function of a product of words depends only on the autocorrelations of the component words.

Lemma 1. Let $B=\left(b_{0}, b_{1}, \ldots, b_{n-1}\right)$ and $A=\left(a_{0}, a_{1}, \ldots, a_{m-1}\right)$ be two words (i.e. blocks over $\mathbb{T}$ ). For each $0 \leq s<n$ and $0 \leq t<m$ we have

$$
\Phi_{B \times A}(s+n t)=\Phi_{B}(s) \Phi_{A}(t)+\overline{\Phi_{B}(n-s)} \Phi_{A}(t+1)
$$

(with the convention that $\Phi_{B}(n)=0$ ).

Remark 1 . The same holds true if $A$ represents a sequence over $\mathbb{T}$ for which the autocorrelation function exists.

Definition 3. Let $\left(B_{1}, B_{2}, \ldots\right)$ be a sequence of blocks over a finite group $G$ such that, for each $q \in \mathbb{N}$,

1) the length $n_{q}$ of $B_{q}$ is at least 2 , and

2) $B_{q}(0)=1$.

The (one-sided, generalized) Morse sequence $A$ determined by the sequence $\left(B_{q}\right)$ of blocks is defined as the coordinatewise limit of the words $A_{q}=$ $B_{1} \times \ldots \times B_{q}$ (convergence is granted by the condition $B_{q}(0)=\mathbf{1}$ ). We exclude periodic sequences from the class of Morse sequences.

We denote by $A^{q}$ the infinite product $B_{q+1} \times B_{q+2} \times \ldots$ Note that $A^{q}$ is also a Morse sequence, and that $A=A_{q} \times A^{q}$. The length of $A_{q}$ (equal to $\left.n_{1} \ldots n_{q}\right)$ is denoted by $m_{q}$.

We remark that the sequence of blocks $\left(B_{q}\right)$ leading to $A$ is not unique. For instance, we can apply "grouping" and define $B_{1}^{\prime}=B_{1} \times \ldots \times B_{q_{1}}$, $B_{2}^{\prime}=B_{q_{1}+1} \times \ldots \times B_{q_{2}}$, etc., to obtain $A=B_{1}^{\prime} \times B_{2}^{\prime} \times \ldots$

Let $A$ be a Morse sequence over $G$. We define $X_{A} \subset G^{\mathbb{Z}}$ as the set of all bi-infinite sequences $x$ such that every block appearing in $x$ appears infinitely many times in $A$. It is clear that $X_{A}$ is closed and $\sigma$-invariant where $\sigma$ is the left shift transformation

$$
(\sigma x)_{n}=x_{n+1} \quad\left(x \in G^{\mathbb{Z}}\right) .
$$


The subshift $\left(X_{A}, \sigma\right)$ will be called the Morse flow generated by $A$ (or by the sequence $\left(B_{1}, B_{2}, \ldots\right)$ of blocks).

In the language of cocycles, the above flow can be described as follows: the base $(X, T, \nu)$ is the rotation of the group of $\left(m_{q}\right)$-adic integers (odometer). We write $T(x)=x+1$. Then, for each $q, X$ decomposes as the disjoint union

$$
X=X_{q} \cup\left(X_{q}+1\right) \cup\left(X_{q}+2\right) \cup \ldots \cup\left(X_{q}+m_{q}-1\right),
$$

where $X_{q}=m_{q} X$ is a closed and open subgroup. The cocycle associated with the Morse sequence $A$ is defined inductively on $X$ as follows:

$$
\phi(x)=A_{q}(i+1)\left(A_{q}(i)\right)^{-1}
$$

for each $x \in X_{q}+i, i=0,1, \ldots, m_{q}-2$. It is elementary to see that the above definition is consistent (i.e., if $\phi(x)$ is defined using $q$ then it will not change for $q^{\prime}>q$ ), and that eventually $\phi$ is defined as a continuous function at all points except at -1 . Moreover, it can be proved that a cocycle $\phi$ defined on an odometer and taking values in a finite abelian group $G$ yields a group extension isomorphic to a Morse flow if and only if $\phi$ has exactly one discontinuity point.

Morse flows have been extensively studied for their dynamical and spectral properties. We refer the reader to $[\mathrm{G}],[\mathrm{J}],[\mathrm{K} 1],[\mathrm{K} 2],[\mathrm{Kw}],[\mathrm{K}-\mathrm{S}],[\mathrm{M}]$. For us it is important to know the following five facts:

FACT 1 (see [I-L] or $[\mathrm{M}]$ for similar statements). A sufficient condition for a Morse flow generated by a sequence of blocks $\left(B_{1}, B_{2}, \ldots\right)$ to be strictly ergodic is that there exist $q_{0} \geq 1$ and $\varepsilon_{A}>0$ such that for each $q \geq q_{0}$,

$$
\operatorname{fr}_{A^{q}}(1, g)>\varepsilon_{A}
$$

for all $g \in G$, where $A^{q}$ denotes the Morse sequence defined by the "truncated" sequence of blocks $\left(B_{q+1}, B_{q+2}, \ldots\right)$.

From now on we assume that our Morse sequence $A$ satisfies the condition of Fact 1. This implies in particular that for every nontrivial character $\gamma \in \widehat{G}$

$$
\underset{q}{\limsup }\left|\Phi_{\gamma\left(A^{q}\right)}(1)\right|<1-\xi_{A} \quad \text { for some } \xi_{A}>0 .
$$

REMARK 2. The above is a natural requirement and in most papers on Morse flows this or similar assumptions are made to ensure ergodicity of the flow and continuity of the interesting part of the spectrum. Our condition also ensures that the flow does not reduce to a Morse flow over a subgroup of $G$. 
For a spectral description of the Morse flow we need to consider the Hilbert space $L^{2}=L^{2}\left(\nu_{A}\right)$, where $\nu_{A}$ is the unique invariant measure on $X_{A}$.

FACT 2 (see $[\mathrm{K}-\mathrm{S}],[\mathrm{M}])$. We have the decomposition

$$
L^{2}=\bigoplus_{\gamma \in \widehat{G}} L_{\gamma}^{2},
$$

where $L_{\gamma}^{2}$ is the $\sigma$-invariant subspace of $L^{2}$ defined by

$$
L_{\gamma}^{2}=\left\{f \in L^{2}: f(g x)=\gamma(g) f(x) \text { for each } g \in G \text { and } x \in X_{A}\right\}
$$

( $g x$ is obtained by multiplying all entries of $x$ by $g$ ). Moreover, the spectrum of $\sigma$ on each $L_{\gamma}^{2}$ is:

- simple,

- discrete if $\gamma=\gamma_{0}$,

- continuous for nontrivial characters.

The spectral type of $\sigma$ on $L_{\gamma}^{2}$ with $\gamma \neq \gamma_{0}$ is the same as that of the spectral measure $\mu_{\left(A, f_{\gamma}\right)}\left(\mu_{(A, \gamma)}\right.$ for short) of the function $f_{\gamma}$ defined by

$$
f_{\gamma}(x)=\gamma\left(x_{0}\right) \text {. }
$$

Recall that the Fourier coefficients of the spectral measure $\mu_{(A, f)}$ of a function $f \in L^{2}$ are

$$
\widehat{\mu}_{(A, f)}(k)=\int z^{k} d \mu_{(A, f)}=\int \bar{f}\left(f \circ \sigma^{k}\right) d \nu_{A} .
$$

If $f=f_{\gamma}$ ( $\gamma$ nontrivial) then, by continuity of $f_{\gamma}$ and unique ergodicity, we can evaluate the integrals by taking averages along the trajectory of an element whose right hand side coincides with $A$. In this manner we obtain the following equalities:

FACT 3. The Fourier coefficients of the spectral measures $\mu_{(A, \gamma)}$ coincide with the autocorrelations of the sequence obtained from $A$ by applying the character $\gamma$ :

$$
\widehat{\mu}_{(A, \gamma)}(k)=\lim _{n \rightarrow \infty} \frac{1}{n} \sum_{i=0}^{n-1} \overline{\gamma(A(i))} \gamma(A(i+k))=\Phi_{\gamma(A)}(k)
$$

for each $k \in \mathbb{N}$ (and the autocorrelation functions exist for each $\gamma \in \widehat{G}$ ).

FACT 4 (see [K2] and [C-N] for similar statements). Let $A$ be a Morse sequence defined by a sequence $\left(B_{1}, B_{2}, \ldots\right)$ of blocks satisfying the assumption of Fact 1. Then, for each nontrivial character $\gamma \in \widehat{G}$ and each $q \in \mathbb{N}$, the measures $\mu_{(A, \gamma)}$ and $\mu_{\left(A^{q}, \gamma\right)}$ are equivalent.

In our study of spectral isomorphisms we will compare the behavior of the Morse flows defined by two Morse sequences, which we denote by $A$ and $A^{\prime}$. In our notation we will use the convention that all letters with a "' refer 
to the objects related to the Morse flow defined by $A^{\prime}$, and corresponding to the objects denoted for $A$ by the same letters without a "I".

Our first remark concerns the discrete spectrum factor:

FACT 5. Let $A$ and $A^{\prime}$ be two Morse sequences over $G$ defined by two sequences of blocks $\left(B_{q}\right)$ and $\left(B_{q}^{\prime}\right)$, respectively. Let $\phi: X \rightarrow G$ and $\phi^{\prime}$ : $X^{\prime} \rightarrow G$ be the corresponding cocycles. If the group extensions are spectrally isomorphic then by appropriate grouping of the blocks $\left(B_{q}\right)$ and $\left(B_{q}^{\prime}\right)$ we can obtain new sequences of blocks $\left(C_{q}\right)$ and $\left(C_{q}^{\prime}\right)$ of common lengths $n_{q}$ and leading to the same Morse sequences $A$ and $A^{\prime}$, respectively.

Proof. This is a classical fact in the theory of Morse cocycles. Since the flows are spectrally isomorphic, they have the same discrete part of spectrum, i.e., that in $L_{\gamma_{0}}^{2}$. This implies that they are extensions over the same odometer $X$. Further, both cocycles have a unique discontinuity at -1 . Thus the cocycle into $G \times G$ defined as $\left(\phi, \phi^{\prime}\right)$ is again a Morse cocycle leading to certain blocks $\left(D_{q}\right)$ of length $n_{q}$. We now let $C_{q}$ and $C_{q}^{\prime}$ be the projections of $D_{q}$ to the first and second coordinate, respectively.

In view of the above fact, when considering spectrally isomorphic Morse flows, we will always assume that the defining blocks have a common structure of lengths, i.e., $n_{q}=n_{q}^{\prime}$ for each $q$.

The following fact can be found in [K2]. Sufficiency of the last condition is an immediate consequence of the fact that the variation distance between orthogonal measures equals 2 .

FACT 6 ([K2]). Let $A$ and $A^{\prime}$ be two Morse sequences with the same structure of lengths. Let $\gamma, \gamma^{\prime} \in \widehat{G}$. Then the spectral measures $\mu_{(A, \gamma)}$ and $\mu_{\left(A^{\prime}, \gamma^{\prime}\right)}$ are either equivalent or orthogonal. They are equivalent if and only if

$$
\left\|\mu_{\left(A^{q}, \gamma\right)}-\mu_{\left(A^{\prime q}, \gamma^{\prime}\right)}\right\| \rightarrow 0,
$$

where $\|\cdot\|$ denotes the variation norm of measures.

As an immediate consequence of Facts 2 and 6 we obtain the following

Theorem 1. The Morse flows defined by $A$ and $A^{\prime}$ are spectrally isomorphic if and only if there exists a permutation $\widehat{\pi}$ of $\widehat{G}$ such that $\widehat{\pi}\left(\gamma_{0}\right)=\gamma_{0}$, and, for each $\gamma \in \widehat{G}, \mu_{(A, \gamma)}$ and $\mu_{\left(A^{\prime}, \widehat{\pi}(\gamma)\right)}$ are equivalent, i.e.,

$$
\left\|\mu_{\left(A^{q}, \gamma\right)}-\mu_{\left(A^{\prime q}, \widehat{\pi}(\gamma)\right)}\right\| \rightarrow 0 .
$$

Spectral isomorphism and autocorrelations. In this section we will show that spectral equivalence between $\mu_{(A, \gamma)}$ and $\mu_{\left(A^{\prime}, \gamma^{\prime}\right)}$ mentioned in Theorem 1 can be tested by checking the autocorrelations of the defining blocks $B_{q}$ and $B_{q}^{\prime}$. The common lengths condition is our standing assumption. 
THEOREM 2. With the assumptions of Fact 1 suppose, for some $\gamma$ and $\gamma^{\prime}$, that $\mu_{(A, \gamma)}$ and $\mu_{\left(A^{\prime}, \gamma^{\prime}\right)}$ are equivalent. Then

$$
\sup _{k \in \mathbb{N}}\left|\Phi_{\gamma\left(A^{q}\right)}(k)-\Phi_{\gamma^{\prime}\left(A^{\prime q}\right)}(k)\right| \rightarrow 0
$$

and

$$
\max _{0 \leq s<n_{q}}\left|\Phi_{\gamma\left(B_{q}\right)}(s)-\Phi_{\gamma^{\prime}\left(B_{q}^{\prime}\right)}(s)\right| \rightarrow 0
$$

as $q$ tends to infinity.

Proof. The statement holds trivially for $\gamma=\gamma^{\prime}=\gamma_{0}$. Assume $\gamma \neq \gamma_{0}$ (then also $\left.\gamma^{\prime} \neq \gamma_{0}\right)$. Set $\varepsilon_{q}=\sup _{k \in \mathbb{N}}\left|\Phi_{\gamma\left(A^{q}\right)}(k)-\Phi_{\gamma^{\prime}\left(A^{\prime q}\right)}(k)\right|$. By Fact 3, we have

$$
\begin{aligned}
\varepsilon_{q} & =\sup _{k \in \mathbb{N}}\left|\widehat{\mu}_{\left(A^{q}, \gamma\right)}(k)-\widehat{\mu}_{\left(A^{\prime q}, \gamma^{\prime}\right)}(k)\right|=\sup _{k \in \mathbb{N}}\left|\int z^{k} d\left(\mu_{\left(A^{q}, \gamma\right)}-\mu_{\left(A^{\prime q}, \gamma^{\prime}\right)}\right)\right| \\
& \leq\left\|\mu_{\left(A^{q}, \gamma\right)}-\mu_{\left(A^{\prime q}, \gamma^{\prime}\right)}\right\| .
\end{aligned}
$$

By the second statement of Fact 6 , we obtain $\varepsilon_{q} \rightarrow 0$, which proves the first convergence. Furthermore, using Remark 1 (after Lemma 1) for $B=\gamma\left(B_{q}\right)$ and $A=\gamma\left(A^{q}\right)$ (then $B \times A=\gamma\left(A^{q-1}\right)$ ) and $t=0$, we have, for every $0 \leq s<n_{q}$,

$$
\begin{aligned}
\Phi_{\gamma\left(A^{q-1}\right)}(s) & =\Phi_{\gamma\left(B_{q}\right)}(s)+\overline{\Phi_{\gamma\left(B_{q}\right)}\left(n_{q}-s\right)} \Phi_{\gamma\left(A^{q}\right)}(1), \\
\Phi_{\gamma\left(A^{q-1}\right)}\left(n_{q}-s\right) & =\Phi_{\gamma\left(B_{q}\right)}\left(n_{q}-s\right)+\overline{\Phi_{\gamma\left(B_{q}\right)}(s)} \Phi_{\gamma\left(A^{q}\right)}(1),
\end{aligned}
$$

from which we obtain

$$
\Phi_{\gamma\left(B_{q}\right)}(s)=\frac{\Phi_{\gamma\left(A^{q-1}\right)}(s)-\Phi_{\gamma\left(A^{q}\right)}(1) \overline{\Phi_{\gamma\left(A^{q-1}\right)}\left(n_{q}-s\right)}}{1-\left|\Phi_{\gamma\left(A^{q}\right)}(1)\right|^{2}}
$$

(by the observation following the statement of Fact 1, for large $q$ the denominator is bounded away from zero). An analogous formula holds for $\gamma^{\prime}$, $B_{q}^{\prime}, A^{\prime q-1}$ and $A^{\prime q}$. Then

$$
\left|\Phi_{\gamma\left(B_{q}\right)}(s)-\Phi_{\gamma^{\prime}\left(B_{q}^{\prime}\right)}(s)\right| \leq \eta_{q},
$$

where $\eta_{q}$ is a function of $\varepsilon_{q-1}, \varepsilon_{q}, \xi_{A}$ and $\xi_{A}^{\prime}$ (not depending on $s$ ) converging to zero. This ends the proof.

Corollary 1. If the Morse flows defined by $A$ and $A^{\prime}$ are spectrally isomorphic and $\widehat{\pi}$ is the permutation as in Theorem 1 then

$$
\begin{array}{r}
\sup _{k \in \mathbb{N}}\left|\Phi_{\gamma\left(A^{q}\right)}(k)-\Phi_{\widehat{\pi}(\gamma)\left(A^{\prime q}\right)}(k)\right| \rightarrow 0, \\
\max _{0 \leq s<n_{q}}\left|\Phi_{\gamma\left(B_{q}\right)}(s)-\Phi_{\widehat{\pi}(\gamma)\left(B_{q}^{\prime}\right)}(s)\right| \rightarrow 0 .
\end{array}
$$

We remark that we have made no effort to reverse our Theorem 2. It is known that even in the case of uniform convergence of Fourier coefficients 
a limit measure may be orthogonal to all measures in the sequence. However, with an additional constraint we have the following result (in fact a strengthening of Theorem 2 in $[\mathrm{Kw}])$ :

TheOREM 3. Suppose, with the assumptions of Fact 1, that the lengths $n_{q}$ are bounded. Then $\mu_{(A, \gamma)}$ and $\mu_{\left(A^{\prime}, \gamma^{\prime}\right)}$ are equivalent for some $\gamma$ and $\gamma^{\prime}$ if and only if there exists $q_{0} \in \mathbb{N}$ such that

$$
\Phi_{\gamma\left(B_{q}\right)} \equiv \Phi_{\gamma^{\prime}\left(B_{q}^{\prime}\right)}
$$

(i.e., $\Phi_{\gamma\left(B_{q}\right)}(s)=\Phi_{\gamma^{\prime}\left(B_{q}^{\prime}\right)}(s)$ for each $\left.0 \leq s<n_{q}\right)$ whenever $q \geq q_{0}$. For such $q$ we have

$$
\mu_{\left(A^{q}, \gamma\right)}=\mu_{\left(A^{\prime q}, \gamma^{\prime}\right)} .
$$

Proof. Sufficiency follows from Facts 3 and 4 . Necessity is an immediate consequence of Theorem 2 and the observation that now there are only finitely many words to choose from. The last statement then follows from Lemma 1 and Fact 3.

Combining Theorem 1 with Theorem 3 we obtain the following characterization of spectral isomorphisms between Morse flows with bounded lengths of defining blocks:

Theorem 4. Let $A$ and $A^{\prime}$ be two Morse sequences over $G$ defined by two sequences of blocks $\left(B_{q}\right)$ and $\left(B_{q}^{\prime}\right)$, respectively, with bounded lengths. Then the Morse flows defined by $A$ and $A^{\prime}$ are spectrally isomorphic if and only if there exists $q_{0} \in \mathbb{N}$ and a permutation $\widehat{\pi}$ of $\widehat{G}$ such that $\widehat{\pi}\left(\gamma_{0}\right)=\gamma_{0}$, $\widehat{\pi}\left(\gamma^{-1}\right)=(\widehat{\pi}(\gamma))^{-1}$, and

$$
\Phi_{\gamma\left(B_{q}\right)} \equiv \Phi_{\widehat{\pi}(\gamma)\left(B_{q}^{\prime}\right)}
$$

for each $\gamma \in \widehat{G}$ and $q \geq q_{0}$.

Proof. Only the property $\widehat{\pi}\left(\gamma^{-1}\right)=(\widehat{\pi}(\gamma))^{-1}$ needs a comment. Obviously, we have $\Phi_{\gamma^{-1}\left(B_{q}\right)}=\bar{\Phi}_{\gamma\left(B_{q}\right)}$. Thus if $\gamma$ and $\widehat{\pi}(\gamma)$ satisfy the displayed formula, then the same holds for $\gamma^{-1}$ and $(\widehat{\pi}(\gamma))^{-1}$. It is now not hard to see that the permutation can be modified to one that satisfies the required condition.

REMARK 3. A question arises: what are the possible pairs of words starting with 1 and having the same autocorrelation functions? Reconstructing a signal from its autocorrelation function is a subject in the field of Information Theory; unfortunately the "signal" has usually a slightly different setup than our "word". There are three natural cases where two words, say $B$ and $B^{\prime}$, have the same autocorrelations:

(a) $B=\left(b_{0}, b_{1}, \ldots, b_{n-1}\right)$ and $B^{\prime}$ is the "flipped word"

$$
B^{*}=\left(b_{0}^{*}, b_{1}^{*}, \ldots, b_{n-1}^{*}\right), \quad \text { where } b_{i}^{*}=\bar{b}_{n-1-i} b_{n},
$$


(b) $B=C \times D$ and $B^{\prime}=C \times D^{*}\left(\right.$ or $\left.B^{\prime}=C^{*} \times D=\left(C \times D^{*}\right)^{*}\right)$,

(c) $B$ is a concatenation of products: $B=\left(C_{1} \times D\right) \ldots\left(C_{k} \times D\right)$, where $C_{1}, \ldots, C_{k}$ have the same length, and $B^{\prime}=\left(C_{1} \times D^{*}\right) \ldots\left(C_{k} \times D^{*}\right)$.

However, using a simple computer program, we have found pairs $B, B^{\prime}$ not being flip of each other and whose length is prime (which eliminates any product representation as in (b) or (c)). We classify such pairs as (d). The shortest such examples are over $\mathbb{Z}_{3}$ and have length 13 :

$$
\begin{aligned}
B & =(1,1,1,1, \bar{p}, \bar{p}, 1,1, p, 1, p, \bar{p}, 1), \\
B^{\prime} & =(1,1,1, \bar{p}, \bar{p}, 1, p, p, p, \bar{p}, p, \bar{p}, 1),
\end{aligned}
$$

and another pair:

$$
\begin{aligned}
C & =(1, p, 1,1, \bar{p}, 1, p, 1, \bar{p}, 1,1, p, 1), \\
C^{\prime} & =(1, p, 1, \bar{p}, \bar{p}, p, \bar{p}, p, \bar{p}, \bar{p}, 1, p, 1)
\end{aligned}
$$

( $p$ denotes the primary root of unity of degree 3 ). The second pair is more interesting because it does not satisfy the condition AS (see Definition 5 below). There also exists an example over $\mathbb{Z}_{2}$ of length 17 .

Regular spectral isomorphisms. Before we specify the advantage of a regular spectral isomorphism between Morse flows, i.e., what kind of "nice" properties we meant in the introduction, we explain how to extend the notion of an autocorrelation to blocks over $G$ without referring to characters. We can temporarily skip the assumption that the group $G$ is finite.

Each compact group $G$ gives rise to a compact simplex $K$ whose extreme points are the elements of $G$ and zero. Classically, the simplex can be obtained as the set of all probability measures on $G \cup\{0\}$, but for us it will be more convenient first to identify $G$ with $\widehat{\widehat{G}}$ (from now on we will thus skip the hat over $g$ and write $g(\gamma))$ and then to let $K$ be the closed convex hull of $G \cup\{0\}$ in the pointwise convergence of functions on $\widehat{G}$. The characters $\gamma$ extend naturally, via $\gamma(\kappa)=\kappa(\gamma)(\kappa \in K)$, to affine functions on $K$ which separate points. Thus, for subconvex combinations we have

$$
\kappa=\sum_{i} \alpha_{i} g_{i} \Longleftrightarrow \forall(\gamma \in \widehat{G}) \gamma(\kappa)=\sum_{i} \alpha_{i} \gamma\left(g_{i}\right)
$$

$\left(\kappa \in K, g_{i} \in G, \alpha_{i} \geq 0, \sum_{i} \alpha_{i} \leq 1\right)$.

We can now define an (abstract) autocorrelation of a block $B$ over $G$ as a subconvex combination of the elements of $G$ with appropriate frequencies as coefficients (subconvex because the coefficients add up to less than 1 - this is why we added an extreme point at zero):

$$
\Phi_{B}(k)=\frac{1}{n} \sum_{i=0}^{n-k-1} \bar{b}_{i} b_{i+k}=\sum_{g \in G} g \operatorname{fr}_{B}(k, g) .
$$


The autocorrelations of sequences over $G$ are defined analogously, as appropriate limits, and they too belong to $K$. By the affine property of characters, we have

$$
\Phi_{\gamma(B)}(k)=\gamma\left(\Phi_{B}(k)\right),
$$

where $B$ denotes either a block or a sequence. Moreover, for two blocks (or sequences) $B, B^{\prime}$ we have

$$
\Phi_{B}(k)=\Phi_{B^{\prime}}(k) \Longleftrightarrow \forall(\gamma \in \widehat{G}) \Phi_{\gamma(B)}(k)=\Phi_{\gamma\left(B^{\prime}\right)}(k) .
$$

REMARK $4 . K$ is a semigroup under multiplication and the extensions of characters are multiplicative functions. This allows us to extend Lemma 1 to blocks (not only words).

We now return to finite groups and Morse flows. Using the above, we immediately see that for regular spectral isomorphisms between Morse flows Corollary 1 and Theorem 4 take on the following form:

COROllary 2. If two Morse flows are regularly spectrally isomorphic then

and

$$
\sup _{k \in \mathbb{N}} \operatorname{dist}\left(\Phi_{A^{q}}(k), \Phi_{v\left(A^{\prime} q\right)}(k)\right) \rightarrow 0
$$

$$
\max _{0 \leq s<n_{q}} \operatorname{dist}\left(\Phi_{B_{q}}(s), \Phi_{v\left(B_{q}^{\prime}\right)}(s)\right) \rightarrow 0
$$

as $q \rightarrow \infty$, where dist denotes the distance in $K$ and $v$ is a group automorphism of $G$.

COROLlaRY 3. Two Morse flows with bounded lengths are regularly spectrally isomorphic if and only if there exists a group automorphism $v$ of $G$ such that

$$
\Phi_{B_{q}} \equiv \Phi_{v\left(B_{q}^{\prime}\right)} \quad \text { for } q \geq q_{0}
$$

The practical meaning of the last statements is that such a spectral isomorphism preserves the limit frequencies:

$$
\max _{0 \leq s<n_{q}}\left|\operatorname{fr}_{B_{q}}(s, g)-\mathrm{fr}_{v\left(B_{q}^{\prime}\right)}(s, g)\right| \underset{q}{\rightarrow} 0
$$

for each $g \in G$, and for bounded lengths we have even more:

$$
\operatorname{fr}_{B_{q}}(k, g)=\operatorname{fr}_{v\left(B_{q}^{\prime}\right)}(k, g)
$$

for each $0 \leq k \leq n-1, g \in G, q \geq q_{0}$. This invariant is of a purely combinatorial nature.

In the remainder of this section we will show that within certain classes of Morse flows every spectral isomorphism is equivalent to a regular one.

The case of a cyclic group of prime order. It will be more convenient to denote the cyclic group additively. Consider the case where $G$ is the cyclic 
group $\mathbb{Z}_{p}$. First observe that if $p \leq 4$ then any permutation preserving zero and negation is an automorphism, and hence every spectral isomorphism between Morse flows over $\mathbb{Z}_{p}$ is regular. But there are stronger reasons why the same must also hold for larger prime numbers $p$.

TheOREM 5. Let $\mathbb{Z}_{p}$ be the (additive) cyclic group of order $p$ where $p>2$ is prime. Consider two Morse sequences $A, A^{\prime}$ over $\mathbb{Z}_{p}$ defined by two sequences of blocks $\left(B_{q}\right)$ and $\left(B_{q}^{\prime}\right)$, with bounded lengths. Then the corresponding Morse flows are spectrally isomorphic if and only if they are regularly spectrally isomorphic (via a group automorphism $v$ ). Moreover, only two cases are possible:

(A) there are infinitely many numbers $q$ for which either $B_{q}$ or $B_{q}^{\prime}$ is not symmetric; then both flows have simple spectrum and the automorphism $v$ is unique,

(B) all blocks $B_{q}$ and $B_{q}^{\prime}$ for $q \geq q_{0}$ are symmetric; then both flows have spectral multiplicity 2 homogeneous on the continuous part. In this case regularity is realized by exactly two group automorphisms, $v$ and $-v$.

REMARK 5 . The fact that Morse flows over $\mathbb{Z}_{p}$ have spectral multiplicity either 1 or 2 generalizes a result of $[\mathrm{K}-\mathrm{S}]$, where the same is obtained with the assumption that the sequence $\left(B_{q}\right)$ is constant.

Proof (of Theorem 5). Note that $\widehat{\mathbb{Z}}_{p}=\mathbb{Z}_{p}$, and each character $\gamma$ on $\mathbb{Z}_{p}$ has the form $\gamma_{s}(r)=\varepsilon^{s r}$, where $\varepsilon$ denotes the primary root of unity of degree $p$, and $r, s \in \mathbb{Z}_{p}$. Theorem 4 says that

$$
\Phi_{\gamma_{s}\left(B_{q}\right)} \equiv \Phi_{\gamma_{\hat{\pi}(s)}\left(B_{q}^{\prime}\right)} \quad \text { for } q \geq q_{0} .
$$

In this notation we have $\widehat{\pi}(0)=0$ and $\widehat{\pi}(-s)=-\widehat{\pi}(s)$. We can also assume that $\widehat{\pi}(1)=1$ (indeed, there exists a group automorphism $w: \mathbb{Z}_{p} \rightarrow \mathbb{Z}_{p}$ sending 1 to $\widehat{\pi}(1)$; the Morse flow generated by $w\left(A^{\prime}\right)$ is spectrally isomorphic to that of $A$ via the permutation $\widehat{\pi}^{\prime}(s)=w^{-1}(\widehat{\pi}(s))$, which clearly satisfies $\left.\widehat{\pi}^{\prime}(1)=1\right)$.

Fix $q \geq q_{0}$ and let $n=n_{q}$ be the length of $B_{q}$. Applying the second part of Definition 2, for each $1 \leq k<n$ and $s \in \mathbb{Z}_{p}$ we can write

$$
\Phi_{\gamma_{s}\left(B_{q}\right)}(k)=\sum_{r \in \mathbb{Z}_{p}} \varepsilon^{s r} \operatorname{fr}_{B_{q}}(k, r), \quad \Phi_{\gamma_{s}\left(B_{q}^{\prime}\right)}(k)=\sum_{r \in \mathbb{Z}_{p}} \varepsilon^{s r} \operatorname{fr}_{B_{q}^{\prime}}(k, r) .
$$

We will prove that

$$
\mathrm{fr}_{B_{q}}(k, r)=\operatorname{fr}_{B_{q}^{\prime}}(k, r)
$$

for every $r \in \mathbb{Z}_{p}$ and every $k$. Consider the following polynomial of degree $p-1$ with rational coefficients:

$$
W(z)=\sum_{0 \leq r \leq p-1} z^{r}\left(\operatorname{fr}_{B_{q}}(k, r)-\operatorname{fr}_{B_{q}^{\prime}}(k, r)\right) .
$$


Since $\widehat{\pi}(1)=1$, we see that $\varepsilon$ is a zero of this polynomial. On the other hand, $W(z)$ has a rational zero at 1 (because the sum of frequencies in each block is equal to $(n-k) / n)$. This yields that either $W(z) \equiv 0$ or $\varepsilon$ is an algebraic number of degree $p-2$. The latter possibility contradicts the well known fact that $\varepsilon$ is an algebraic number of degree $p-1$.

Having derived equality of the corresponding frequencies in $B_{q}$ and $B_{q}^{\prime}$, we now see that $\Phi_{\gamma_{s}\left(B_{q}\right)}(k)=\Phi_{\gamma_{s}\left(B_{q}^{\prime}\right)}(k)$ for every $s$, which implies that the identity permutation $\widehat{\pi}=$ id (which obviously is a group automorphism) establishes regularity of the given or an equivalent spectral isomorphism.

In order to prove the second part of the theorem, first assume that the identity is a unique such permutation. Then we conclude that for infinitely many indices $q \geq q_{0}$ both blocks $B_{q}, B_{q}^{\prime}$ are asymmetric (if for each $q \geq q_{0}$ at least one of $B_{q}, B_{q}^{\prime}$ is symmetric then this block, say $B$, has the same autocorrelations as $-B$, hence the permutation - id provides a second possibility). Moreover, both flows then have simple spectrum, because otherwise there would again be more than one permutation. Thus we have the situation as in assertion (A).

Suppose that another permutation $\widehat{\pi}$ is possible. Let

$$
B_{q}=\left(\varepsilon^{r_{0}}, \varepsilon^{r_{1}}, \ldots, \varepsilon^{r_{n-1}}\right) .
$$

For each $s$ we have $\Phi_{\gamma_{s}\left(B_{q}\right)} \equiv \Phi_{\gamma_{\hat{\pi}(s)}\left(B_{q}^{\prime}\right)}$, but also, as proved before, $\Phi_{\gamma_{s}\left(B_{q}\right)} \equiv$ $\Phi_{\gamma_{s}\left(B_{q}^{\prime}\right)}$. Hence, we obtain

$$
\Phi_{\gamma_{s}\left(B_{q}\right)} \equiv \Phi_{\gamma_{\hat{\pi}(s)}\left(B_{q}\right)} .
$$

Let $k$ be the smallest index for which at least one of the exponents $r_{k}, r_{n-1-k}$ is different from 0. For simplicity of notation, we set $a=r_{k}, b=r_{n-1-k}$. We have

$$
\begin{aligned}
\Phi_{\gamma_{s}\left(B_{q}\right)}(n-1-k) & =\frac{1}{n}\left(\varepsilon^{b s}+\varepsilon^{-a s}\right), \\
\Phi_{\gamma_{\widehat{\pi}(s)}\left(B_{q}\right)}(n-1-k) & =\frac{1}{n}\left(\varepsilon^{b \widehat{\pi}(s)}+\varepsilon^{-a \widehat{\pi}(s)}\right) .
\end{aligned}
$$

We use the following elementary fact concerning unimodular complex numbers: if $z_{1}+z_{2}=z_{3}+z_{4} \neq 0$ then either $\left(z_{1}=z_{3}\right.$ and $\left.z_{2}=z_{4}\right)$ or $\left(z_{1}=z_{4}\right.$ and $\left.z_{2}=z_{3}\right)$. Since no two roots of unity of degree $p$ add to zero, we must have either

$$
\begin{aligned}
b s & =b \widehat{\pi}(s) & \text { and } & a s=a \widehat{\pi}(s), \quad \text { or } \\
b s & =-a \widehat{\pi}(s) & \text { and } & a s=-b \widehat{\pi}(s) .
\end{aligned}
$$

Since either $a$ or $b$ is nonzero, and $\mathbb{Z}_{p}$ is a field, we have $\widehat{\pi}(s)=s$ in the first case, and $(\widehat{\pi}(s))^{2}=s^{2}$ in the second. The latter equation has in $\mathbb{Z}_{p}$ two solutions $\widehat{\pi}(s)= \pm s$. 
Since $\widehat{\pi} \neq \mathrm{id}, \widehat{\pi}(s)=-s$ is valid for some $s \neq 0$. Then

$$
\Phi_{\gamma_{s}\left(B_{q}\right)} \equiv \Phi_{\gamma_{-s}\left(B_{q}\right)} .
$$

We now prove that $B_{q}$ is symmetric. Suppose the converse, and let $k$ be the smallest index for which $a=r_{k} \neq b=r_{n-1-k}$. We have

$$
\begin{gathered}
\Phi_{\gamma_{s}\left(B_{q}\right)}(n-1-k)=\frac{1}{n}\left(\varepsilon^{b s}+R+\varepsilon^{-a s}\right), \\
\Phi_{\gamma_{-s}\left(B_{q}\right)}(n-1-k)=\frac{1}{n}\left(\varepsilon^{-b s}+\bar{R}+\varepsilon^{a s}\right),
\end{gathered}
$$

where $R$ denotes the part of the formula involving the remaining (outer) terms of the block. But, by symmetry of the outer part of $B_{q}, R$ is a real number, hence, as before, either

$$
\begin{array}{rll}
b s=-b s & \text { and } & a s=-a s, \quad \text { or } \\
b s=a s & \text { and } & -a s=-b s .
\end{array}
$$

In either case $a=b$, a contradiction.

The fact that in the symmetric case the spectral multiplicity is not larger than 2 is now obvious: in the converse case there would exist a third permutation. This completes the proof of (B).

Strongly asymmetric blocks. In the case of a general finite group $G$ we can prove that every spectral isomorphism between Morse flows is regular under some additional assumptions on the structure of the defining blocks $\left(B_{q}\right)$ (we need them to be nonsymmetric in a stronger sense). First we make the following observations:

Let $\widehat{\pi}$ be any permutation of $\widehat{G}$. Then $\widehat{\pi}$ can be viewed as a Haar measure preserving transformation on $\widehat{G}$. Thus it induces a unitary operator on $L^{2}(\widehat{G})$. The characters on $\widehat{G}$ form an orthogonal base in $L^{2}(\widehat{G})$ and they have the form $\widehat{g}(\gamma)=\gamma(g)(g \in G)$. Developing their images $\widehat{g} \circ \widehat{\pi}$ in the base we obtain

$$
\widehat{g} \circ \widehat{\pi}=\sum_{h \in G} \Pi(h, g) \widehat{h},
$$

where $\Pi$ is some complex square matrix with rows and columns indexed by the elements of $G$.

Lemma 2. Let $\widehat{\pi}$ be the permutation of $\widehat{G}$ as in Theorem 4. The matrix $\Pi$ has the following properties:

(i) $\Pi$ is unitary,

(ii) $\Pi\left(h, g_{1} g_{2}\right)=\sum_{e \in G} \Pi\left(e, g_{1}\right) \Pi\left(h e^{-1}, g_{2}\right)$ for any $h, g_{1}, g_{2} \in G$,

(iii) $\Pi(\mathbf{1}, \mathbf{1})=1, \Pi(\mathbf{1}, h)=\Pi(h, \mathbf{1})=0$ if $h \neq \mathbf{1}$,

(iv) $\Pi\left(h, g^{-1}\right)=\Pi\left(h^{-1}, g\right)$ for any $h, g \in G$. 
Proof. The properties (i)-(ii) follow from the fact that $\widehat{\pi}$ represents a unitary and multiplicative operator on $L^{2}(\widehat{G})$. The statements (iii) and (iv) can be easily derived from the conditions $\widehat{\pi}\left(\gamma_{0}\right)=\gamma_{0}$ and $\widehat{\pi}\left(\gamma^{-1}\right)=(\widehat{\pi}(\gamma))^{-1}$. We omit the detailed calculations.

Lemma 3. Let $A$ and $A^{\prime}$ be as in Theorem 4 and suppose the corresponding Morse flows are spectrally isomorphic. Then for every $q \geq q_{0}$, $0 \leq k<n_{q}$ and $h \in G$ we have

$$
\mathrm{fr}_{B_{q}^{\prime}}(k, g)=\sum_{h \in G} \overline{\Pi(h, g)} \mathrm{fr}_{B_{q}}(k, h) .
$$

Proof. Write for short $B=B_{q}, B^{\prime}=B_{q}^{\prime}$ and $n=n_{q}$. By Theorem 4, we have

$$
\Phi_{\gamma(B)} \equiv \Phi_{\widehat{\pi}(\gamma)\left(B^{\prime}\right)}
$$

i.e., for every fixed $0 \leq k<n$,

$$
\sum_{h \in G} \gamma(h) \mathrm{fr}_{B}(k, h)=\sum_{g \in G} \widehat{\pi}(\gamma)(g) \mathrm{fr}_{B^{\prime}}(k, g) .
$$

The right hand side can be rewritten as $\sum_{g \in G}(\widehat{g} \circ \widehat{\pi})(\gamma) \operatorname{fr}_{B^{\prime}}(k, g)$. By the formula $(*)$ we then obtain

$$
\begin{aligned}
\sum_{h \in G} \gamma(h) \mathrm{fr}_{B}(k, h) & =\sum_{g \in G} \sum_{h \in G} \Pi(h, g) \widehat{h}(\gamma) \mathrm{fr}_{B^{\prime}}(k, g) \\
& =\sum_{h \in G} \gamma(h) \sum_{g \in G} \Pi(h, g) \mathrm{fr}_{B^{\prime}}(k, g)
\end{aligned}
$$

(the equalities hold for each $\gamma \in \widehat{G}$ ). Viewing the above as a function of the variable $\gamma \in \widehat{G}$ and by uniqueness of the representation in the basis $\{\widehat{h}: h \in G\}$ we obtain $\operatorname{fr}_{B}(k, h)=\sum_{g \in G} \Pi(h, g) \operatorname{fr}_{B^{\prime}}(k, g)$ for each $h$. Since $\Pi$ is unitary, $\Pi^{-1}=\overline{\Pi^{T}}$, and the desired equality holds.

Suppose that $\Pi(h, g)=1$ for some $h, g \in G$. Then, by (i), $\Pi(e, g)=0$ if $e \neq h$ and $\Pi(h, e)=0$ if $e \neq g$.

Definition 4. We say that $g \in G$ has the permutation property if $\Pi(h, g)=1$ for some $h \in G$. Then we denote by $v(g)$ the element $h$ so determined.

Lemma 4. The set $G_{v}$ of those $g \in G$ which have the permutation property is a subgroup, and $v$ is a group isomorphism between $G_{v}$ and its image by $v$.

Proof. By (iii), $\mathbf{1} \in G_{v}$, and by (iv), $g \in G_{v} \Rightarrow g^{-1} \in G_{v}$. It suffices to check that $g_{1}, g_{2} \in G_{v} \Rightarrow g_{1} g_{2} \in G_{v}$ and $v\left(g_{1} g_{2}\right)=v\left(g_{1}\right) v\left(g_{2}\right)$. Indeed, 
by (ii),

$$
\Pi\left(h, g_{1} g_{2}\right)=\sum_{e \in G} \Pi\left(e, g_{1}\right) \Pi\left(h e^{-1}, g_{2}\right) .
$$

By the permutation property of $g_{1}$ and $g_{2}$, the only nonzero summand is where $e=v\left(g_{1}\right)$ and simultaneously $h e^{-1}=v\left(g_{2}\right)$. Since a nonzero summand exists for some $h$, we obtain $h=v\left(g_{1}\right) v\left(g_{2}\right)$. By uniqueness of such $h$, $\Pi\left(h, g_{1} g_{2}\right)=1$, hence $g_{1} g_{2} \in G_{v}$ and $v\left(g_{1} g_{2}\right)=v\left(g_{1}\right) v\left(g_{2}\right)$, as needed.

We will prove regularity of spectral isomorphism between Morse sequences whose defining blocks $B_{p}$ satisfy a certain condition of asymmetry. For a given block $B=\left(b_{0}, b_{1}, \ldots, b_{n-1}\right) \in G^{n}$ and $0 \leq k \leq n / 2$ we denote by $G_{k}^{B}$ the subgroup of $G$ generated by the elements $b_{0}, b_{1}, \ldots, b_{k-1}, b_{k}$ and their symmetric counterparts $b_{n-1}, b_{n-2}, \ldots, b_{n-k}, b_{n-k-1}$. If $k>n / 2$ then we put $G_{k}^{B}=G_{k-1}^{B}$.

Definition 5. We say that a sequence of blocks $\left(B_{q}\right)$ has the property $A S$ if for each $k \in \mathbb{N}$ and $q \in \mathbb{N}$ at least one of the elements $b_{k}, b_{n-k-1}$ of $B_{q}$ belongs to the group $G_{k-1}$ generated by $\bigcup_{q} G_{k-1}^{B_{q}}$.

REMARK 6. The above class of sequences of blocks is quite large. For example, in cyclic groups it suffices that the last term of at least one block is a generator.

TheOREM 6. Let $A$ and $A^{\prime}$ be a pair of spectrally isomorphic Morse sequences over $G$, with bounded lengths. The number $q_{0}$ is thus defined (see Theorem 4). If $\left(B_{q}^{\prime}\right)_{q \geq q_{0}}$ (or $\left(B_{q}\right)_{q \geq q_{0}}$ ) has the property $A S$ then $\widehat{\pi}$ is a group automorphism.

Pr o o f. Suppose $\left(B_{q}^{\prime}\right)_{q \geq q_{0}}$ has the property AS. We will inductively prove that $G_{k}^{\prime} \subset G_{v}$ and that $v\left(G_{k}^{\prime}\right)=G_{k}$. Since $\bigcup_{k \in \mathbb{N}} G_{k}^{\prime}=G$, the automorphism $v$ will eventually extend to the whole group $G$.

SteP 0. Fix $q \geq q_{0}$ and set $B=B_{q}, B^{\prime}=B_{q}^{\prime}$ and $n=n_{q}$. By Theorem 4 , for every $\gamma \in \widehat{G}$ we have $\Phi_{\gamma(B)}(n-1)=\Phi_{\widehat{\pi}(\gamma)\left(B^{\prime}\right)}(n-1)$. By the definition of autocorrelations this simply means that

$$
\gamma\left(b_{n-1}\right)=\widehat{\pi}(\gamma)\left(b_{n-1}^{\prime}\right) \quad \text { for each } \gamma,
$$

i.e., that $\widehat{b}_{n-1}^{\prime} \circ \widehat{\pi}=\widehat{b}_{n-1}$. By the formula $(*)$ we obtain

$$
\Pi\left(b_{n-1}, b_{n-1}^{\prime}\right)=1 .
$$

We have proved that $b_{n-1}^{\prime}$ has the permutation property, and $v\left(b_{n-1}^{\prime}\right)=$ $b_{n-1}$. Recall that $b_{0}^{\prime}=b_{0}=\mathbf{1}$, hence $v\left(b_{0}^{\prime}\right)=b_{0}$. We do that for each $q \geq q_{0}$. The application of Lemma 4 yields $G_{0}^{\prime} \subset G_{v}$ and $v\left(G_{0}^{\prime}\right)=G_{0}$. 
STEP $k$. Suppose the statement has been proved for $k-1$. As before, fix $q \geq q_{0}$ and define $B=B_{q}, B^{\prime}=B_{q}^{\prime}$ and $n=n_{q}$. For every $\gamma \in \widehat{G}$ we have

$$
\Phi_{\gamma(B)}(n-k-1)=\Phi_{\widehat{\pi}(\gamma)\left(B^{\prime}\right)}(n-k-1),
$$

i.e.,

$$
\sum_{g \in G} \gamma(g) \operatorname{fr}_{B}(n-k-1, g)=\sum_{g \in G} \widehat{\pi}(\gamma)(g) \operatorname{fr}_{B^{\prime}}(n-k-1, g) .
$$

The above can be written as

$$
\begin{aligned}
L & =\sum_{g \in G_{k-1}} \gamma(g) \operatorname{fr}_{B}(n-k-1, g)+\sum_{g \notin G_{k-1}} \gamma(g) \operatorname{fr}_{B}(n-k-1, g) \\
& =\sum_{g \in G_{k-1}^{\prime}} \widehat{\pi}(\gamma)(g) \operatorname{fr}_{B^{\prime}}(n-k-1, g)+\sum_{g \notin G_{k-1}^{\prime}} \widehat{\pi}(\gamma)(g) \operatorname{fr}_{B^{\prime}}(n-k-1, g)=R .
\end{aligned}
$$

Note that if $g \in G_{v}$ then, by $(*), \widehat{g} \circ \widehat{\pi}=\widehat{v(g)}$, and, by Lemma $3 \operatorname{fr}_{B^{\prime}}(k, g)=$ $\mathrm{fr}_{B}(k, v(g))$. Thus the first sum of $R$ becomes

$$
\sum_{g \in G_{k-1}^{\prime}} \gamma(v(g)) \operatorname{fr}_{B}(n-k-1, v(g))
$$

which, by the assumption that $v\left(G_{k-1}^{\prime}\right)=G_{k-1}$, equals the first sum of $L$. By the property AS, the second sum of $R$ consists of at most one summand: either $n^{-1} \widehat{\pi}(\gamma)\left(b_{n-k-1}^{\prime}\right)$ or $n^{-1} \widehat{\pi}(\gamma)\left(b_{k}^{\prime-1} b_{n-1}^{\prime}\right)$; this is best seen if $R$ is written as

$$
\frac{1}{n} \widehat{\pi}(\gamma)\left(b_{n-k-1}^{\prime}\right)+\frac{1}{n} \sum_{i=1}^{k-1} \widehat{\pi}(\gamma)\left(b_{i}^{\prime-1} b_{i+n-k-1}^{\prime}\right)+\frac{1}{n} \widehat{\pi}(\gamma)\left(b_{k}^{\prime-1} b_{n-1}^{\prime}\right),
$$

because all elements $b_{i}^{\prime-1} b_{i+n-k-1}^{\prime}$ in the central sum belong by definition to $G_{k-1}^{\prime}$. If we let $\gamma=\gamma_{0}$ in the expressions $L$ and $R$, their first sums become the sums of the corresponding frequencies over $G_{k-1}^{\prime}$ and $G_{k-1}$, so, by the previous argument, their common value is either 1 or $1-1 / n$. It is now seen that the second sum of $L$ has as many summands as that of $R$, i.e., zero or one. The case of zero summands is trivial. Suppose we have one summand on each side, say $n^{-1} \widehat{\pi}(\gamma)(g)$ and $n^{-1} \gamma(h)$. Then $\widehat{\pi}(\gamma)(g)=\gamma(h)$ for each $\gamma$. As before, by the formula $(*)$ we obtain $\Pi(h, g)=1$. We have proved that $g \in G_{v}$, and $v(g)=h$. We do that for each $q \geq q_{0}$. So the elements $g_{q}$ obtained exhaust all new elements generating $G_{k}^{\prime}$ and $G_{k}$ ("new" means not in $G_{k-1}^{\prime}$ ), and the $h_{q}$ exhaust all new elements generating $G_{k}$. An application of Lemma 4 yields $G_{k}^{\prime} \subset G_{v}$ and $v\left(G_{k}^{\prime}\right)=G_{k}$.

Morse flows of rank one. Rank is a very important metric invariant and its definition involving towers is commonly known. For symbolic flows 
there exists an equivalent definition in terms of blocks for which we will need some additional notions.

The $\bar{d}$-distance between blocks $B=\left(b_{0}, b_{1}, \ldots, b_{n-1}\right), C=\left(c_{0}, c_{1}, \ldots, c_{n-1}\right)$ (of the same length) is defined by

$$
\bar{d}(B, C)=\frac{1}{n} \#\left\{0 \leq i<n: b_{i} \neq c_{i}\right\} .
$$

Let $A$ be a sequence over $G$. We say that a finite collection $\left\{C_{1}, \ldots, C_{r}\right\}$ of blocks (not necessarily of the same length) $\varepsilon$-covers $A$ if $A$ can be represented as a concatenation $H_{1} A_{1} H_{2} A_{2} \ldots$ such that:

a) $\forall(j \geq 1) \exists(0 \leq i \leq r) \bar{d}\left(A_{j}, C_{i}\right) \leq \varepsilon$, and

b) the blocks $H_{j}$ occupy in $A$ a portion of upper density at most $\varepsilon$ (the blocks $H_{j}$ are referred to as gaps in the $\varepsilon$-covering; a gap is allowed to be the null block).

Definition. If $A$ generates a strictly ergodic subshift $\left(X_{A}, \sigma\right)$ then its rank $r\left(X_{A}, \sigma\right)$ is defined as the smallest integer $r$ such that for every $\delta>0$ there exists a collection $\left\{C_{(\delta, 1)}, \ldots, C_{(\delta, r)}\right\}$ of $r$ blocks, of length at least $1 / \delta$ each, which $\delta$-covers $A$. If no finite $r$ satisfies the above then we set $r\left(X_{A}, \sigma\right)=\infty$.

We remark that 0-1 Morse sequences with rank one were first noticed to exist by Mariusz Lemańczyk ([L]).

Throughout this section we no longer assume the bounded lengths property.

TheOREM 7. Let $A$ be a Morse sequence over a finite group $G$ of order $p$. Then the corresponding Morse flow, $\left(X_{A}, \sigma\right)$, is of rank one if and only if for every $g \in G$ there exists a sequence of integers $k_{n}^{g}$ such that

$$
\widehat{\mu}_{(A, \gamma)}\left(k_{n}^{g}\right) \underset{n \rightarrow \infty}{\longrightarrow} \gamma(g)
$$

for every character $\gamma$ on $G$, i.e., $\Phi_{A}\left(k_{n}^{g}\right) \rightarrow g$ in the simplex $K$.

Proof. The last condition implies that $\operatorname{fr}_{A}\left(k_{n}^{g}, g\right) \rightarrow 1$. For fixed $\delta$ we let $k^{g}$ be such that

$$
\operatorname{fr}_{A}\left(k^{g}, g\right)>1-\delta .
$$

Letting $k=\max \left\{\left|k^{g}\right|: g \in G\right\}$ we now find $q \geq 0$ for which the length $m_{q}$ of $A_{q}$ is larger than $2 k / \delta$ and

$$
\operatorname{fr}_{A_{q}}\left(k^{g}, g\right)>1-\delta
$$

for each $g \in G$. Directly from the definitions of the frequencies and of the $\bar{d}$-distance we obtain

$$
\bar{d}\left(A_{q}\left[k, m_{q}-k\right), g A_{q}\left[k-k^{g}, m_{q}-k-k^{g}\right)\right)<\delta .
$$


Since the Morse sequence $A$ is a concatenation of blocks of the form $g A_{q}$ $(g \in G)$, and $2 k / m_{q}<\delta$, we have just shown how to $\delta$-cover $A$ using copies of the single block $A_{q}\left[k, m_{q}-k\right)$.

The converse implication follows from J. King's Weak Closure Theorem ([Ki] $)$. Indeed, for fixed $g \in G$ consider the transformation $\widetilde{g}: X_{A} \rightarrow X_{A}$ given by $\widetilde{g}(x)=g^{-1} x$, which obviously belongs to the centralizer of the shift transformation $\sigma$ acting on $\left(X_{A}, \nu_{A}\right)$. By the quoted theorem, there exists a sequence of integers $k_{n}=k_{n}^{g}(n \in \mathbb{N})$ such that $\sigma^{k_{n}} \rightarrow \widetilde{g}$ weakly. Replacing $g$ by $g^{-1}$ if necessary, we can assume that $k_{n} \rightarrow \infty$. By Lemma 1.3 of [Ki], we have

$$
\bar{d}\left(\sigma^{k_{n}}(x), g^{-1} x\right) \rightarrow 0
$$

for $\nu_{A}$-almost every $x$. For each $q \geq 1$ every $x$ is a concatenation of the blocks $h A_{q}(h \in G)$, hence there exists a sequence $q_{n}$ with

$$
d_{n}=\bar{d}\left(A_{q_{n}}\left[k_{n}, m_{q_{n}}\right), g^{-1} A_{q_{n}}\left[0, m_{q_{n}}-k_{n}\right)\right) \rightarrow 0 .
$$

By the definitions of $\bar{d}$ and $\mathrm{fr}_{B}$, we have $d_{n}=1-\mathrm{fr}_{A_{q_{n}}}\left(k_{n}, g\right)$. Since $q_{n}$ can be chosen much larger than $k_{n}$, the convergence $\mathrm{fr}_{A}\left(k_{n}, g\right) \rightarrow 1$ follows easily. This implies that for each character $\gamma \in \widehat{G}$, we have $\mu_{(A, \gamma)}\left(k_{n}\right) \rightarrow \gamma(g)$, which completes the proof.

The above theorem has many equivalent versions involving the blocks and sequences $A_{q}, B_{q}$, and $A^{q}$. We shall need the following:

TheOREM 8. The Morse flow $\left(X_{A}, \sigma\right)$ is of rank one if and only if for every $g \in G$ there exists a sequence of integers $\left(k_{q}^{g}\right)_{q}$ such that

$$
\Phi_{A^{q}}\left(k_{q}^{g}\right) \underset{q}{\rightarrow} g \quad \text { in } K .
$$

Proof. Recall that $A=A_{q} \times A^{q}$, where the length of $A_{q}$ is denoted by $m_{q}$. By Lemma 1 (and Remark 4) we obtain

$$
\Phi_{A}\left(m_{q} k_{q}^{g}\right)=\Phi_{A^{q}}\left(k_{q}^{g}\right) \rightarrow g,
$$

which implies sufficiency.

By the definition of rank one it is not hard to see that if $A$ generates a flow of rank one then the same holds for each $A^{q}$. Thus appropriate sequences $\left(k_{n}^{g}\right)$ exist for each $A^{q}$, as in Theorem 7 . The sequence $\left(k_{q}^{g}\right)$ is then chosen diagonally.

We are in a position to state a result which concludes our investigations:

TheOREM 9. If two Morse flows $\left(X_{A}, \sigma\right)$ and $\left(X_{A^{\prime}}, \sigma\right)$ are spectrally isomorphic, and one of them is of rank one, then the corresponding spectral isomorphism is regular and the other flow is also of rank one. 
Proof. Suppose that $\left(X_{A}, \sigma\right)$ is of rank one. By Theorem 8 we have

$$
\Phi_{A^{q}}\left(k_{q}^{g}\right) \rightarrow g,
$$

and by Corollary 1,

$$
\left|\Phi_{\gamma\left(A^{q}\right)}\left(k_{q}^{g}\right)-\Phi_{\widehat{\pi}(\gamma)\left(A^{\prime q}\right)}\left(k_{q}^{g}\right)\right| \rightarrow 0 .
$$

Substituting $\pi^{-1}(\gamma)$ for $\gamma$ we conclude that

$$
\Phi_{A^{\prime} q}\left(k_{q}^{g}\right) \rightarrow g \circ \widehat{\pi}^{-1} .
$$

We have shown that $g \circ \widehat{\pi}^{-1}$ (a function on $\widehat{G}$ ) belongs to the compact set $K$. It is elementary to see that, being unimodular, it is then a nonzero extreme point in $K$. Thus, $g \circ \widehat{\pi}^{-1}=g^{\prime} \in G$. Assigning $v(g)=g^{\prime}$ we obtain $\pi^{-1}(\gamma)=\gamma \circ v$ for each $\gamma$. This $v$ is clearly a group automorphism, hence regularity is proved. We can now replace $g$ by $v^{-1}(g)$ to obtain

$$
\Phi_{A^{\prime} q}\left(k_{q}^{v^{-1}(g)}\right) \rightarrow g,
$$

hence $A^{\prime}$ satisfies the condition of Theorem 8 with ${k^{\prime}}_{q}^{g}=k_{q}^{v^{-1}(g)}$. It follows that $\left(X_{A^{\prime}}, \sigma\right)$ is of rank one, as desired.

\section{References}

[C-N] J. R. Choksi and M. G. Nadkarni, The maximal spectral type of a rank one transformation, Canad. Math. Bull. 37 (1994), 29-36.

[D-L] T. Downarowicz and Y. Lacroix, Merit factors and Morse sequences, Theoret. Comput. Sci. 209 (1998), 377-387.

[G] M. Guenais, Morse cocycles and simple Lebesgue spectrum, Ergodic Theory Dynam. Systems 19 (1999), 437-446.

[J] A. del Junco, A transformation with simple spectrum which is not rank one, Canad. J. Math. 29 (1977), 653-663.

[J-L-M] A. del Junco, M. Lemańczyk and M. Mentzen, Semisimplicity, joinings and group extensions, Studia Math. 112 (1995), 141-164.

[I-L] A. Iwanik and Y. Lacroix, Some constructions of strictly ergodic non-regular Toeplitz flows, ibid. 110 (1994), 191-203.

[K1] M. S. Keane, Generalized Morse sequences, Z. Wahrsch. Verw. Gebiete 10 (1968) 335-353.

[K2] -, Strongly mixing g-measures, Invent. Math. 16 (1972), 309-324.

[Ki] J. King, The commutant is the weak closure of the powers, for rank-1 transformations, Ergodic Theory Dynam. Systems 6 (1986), 363-384.

$[\mathrm{Kw}]$ J. Kwiatkowski, Spectral isomorphism of Morse dynamical systems, Bull. Acad. Polon. Sci. 29 (1981), 105-114.

[K-S] J. Kwiatkowski and A. Sikorski, Spectral properties of G-symbolic Morse shifts, Bull. Soc. Math. France 115 (1987), 19-33.

[L] M. Lemańczyk, The rank of regular Morse dynamical systems, Z. Wahrsch. Verw. Gebiete 70 (1985), 33-48.

[M] J. C. Martin, The structure of generalized Morse minimal sets on n-symbols, Proc. Amer. Math. Soc. 2 (1977), 343-355. 
[N] D. Newton, On canonical factors of ergodic dynamical systems, J. London Math. Soc. 19 (1979), 129-136.

Institute of Mathematics Wrocław University of Technology Wybrzeże Wyspiańskiego 27 50-370 Wrocław, Poland

E-mail: downar@im.pwr.wroc.pl

Département de Mathématiques

Faculté des Sciences et Techniques

Université de Bretagne Occidentale

6 Av. V. Le Gorgeu, B.P. 809

29285 Brest Cedex, France

E-mail: lacroix@univ-brest.fr
Faculty of Mathematics and Informatics Nicholas Copernicus University

Chopina 12/18

87-100 Toruń, Poland

E-mail: jkwiat@mat.uni.torun.pl

Received 7 December 1998;

in revised form 4 May 1999 and 7 December 1999 